\title{
TRANSFORMATION OF ART OBJECTS IN THE 3D DESIGN PROCESS OF
} SHOE PARTS

\author{
MARYNA LESHCHYSHYN, SVITLANA GARKAVENKO, VICTORIA KERNESH, \\ BORYS ZLOTENKO, TETIANA KULIK, ANASTASIA GLIONIK \\ Kyiv National University of Technology and Design, Ukraine, marfiichuk@gmail.com, \\ garkavenko.s@knutd.com.ua,kievfoot@ukr.net, zlotenko.bm@knutd.com.ua, \\ kulik.ti@knutd.com.ua,gloynik.nastia@gmail.com
}

\begin{abstract}
The analysis and classification of shaping methods in the design of shoe bottom parts, and the investigation of research methods of automation of the design process of shaping shoe bottom parts to increase the efficiency of the design process and reduce the complexity of manufacturing products at the stages of technological preparation. Based on the use of means of transformation and harmonization, the structure of the stages of shaping of art objects to obtain modern shapes was developed, which made it possible to implement a new approach to the artistic design of footwear. On the basis of the developed approaches to the choice of shapes and shaping methods sketches were created and 3D models of shoe bottom parts with the subsequent program analysis of loading on a sole were developed. New shapes shoe bottom parts have been developed with the help of transformation principles and a model has been made by automated design methods, which can be used in technological preparation for the manufacture of light industry products. Regularities of transformation processes for improvement of artistic design of footwear on the principles of bionics and stylization are established. Given that the complexity of structures and the emergence of new spatial solutions pose designers more complex technical tasks for the implementation of design projects, a method was proposed of calculating the strength and stability of products of complex geometric shapes, taking into account the materials from which they are made. As a result of the performed work, women's shoes of the "shuttle" design with an over seam back allowance were developed. Given the fact that the shoes should be comfortable and meet ergonomic indicators, the materials, the shape of the pad, and the design of the model were selected. The purpose of this shoe is everyday wearing, focused on the youth category of consumers. The used technique allows shaping a wide range of various prototypes of footwear, using a small number of transformed products. Determining the most loaded areas made it possible to propose measures to improve the design of shoes of non-standard models. And the combination of the given techniques with modern high-tech production helps to save means of the manufacturer and to increase the service life of footwear.
\end{abstract}

Key words: footwear, shaping, transformation.

\section{INTRODUCTION}

At all times, footwear was a necessity, a means of protecting the human foot from the adverse effects of the environment. During thousands of years of its existence, shoe production has undergone significant changes: from hand-made by individual craftsmen to mass production of large batches of the same type of shoes in large factories.

One of the key factors in shaping the modern footwear market is the impact of fashion trends that are changing very fast, especially for women's shoes. The assortment of women's shoes has the tendency to constantly change and update compared to men's. The main factors for this are the change of the season, fashion trends, the emergence of new materials and improvements in production technologies, as well as the constant need of women to change the assortment depending on the psychological feeling. Therefore, women usually do more purchases in the season than men (https://buklib.net/books/28806).

The trends of modern fashion popularize footwear models of complex shapes, which requires the use of non-traditional methods and innovative design technologies. Properly organized three-dimensional structure and a pronounced transformation of the shape of the product create the preconditions for the integrity and harmony of the shape of the shoe. 


\section{SETTING OBJECTIVES}

Design today is advancing within the framework of global large-scale trends that correct everyday concepts. This is expressed in the methods of non-standard shaping, as well as the introduction of new technological materials in combination with color palettes, the result of which guarantees the achievement of artistic expressiveness of the shape of products. Shoes, as a three-dimensional structure, are a complex system of parts that are placed in a specific compositional relationship.

Despite the variety of existing solutions in the field of shaping, today there is a need for research and search for new promising developments in shoe design. Today, 3D technologies are gaining wide popularity in various industries, and their use in the design and manufacture of footwear will be promising. The latest solutions allow improving the transmission of information and design of shoe parts using CAD software, simplifying the process of models designing and reducing the complexity of production.

\section{RESEARCH RESULTS}

Among the main methods of shaping are: combinatorial shaping, transformation, stylization, the method of shaping the form based on a natural analogue (bionics), color shaping (Ustin, 2007; Zaeva-Burdonskaya and Kurasov, 2008).

The richest source of inspiration for the designer is wildlife, which is the basis for finding a decorative combinatorial element. When appealing to nature, the search for a harmonious interaction of content (biological function of the organism), form (the way of existence of the organism) and material (of which the organism itself is composed) is assumed. The shape of a unified element creates a certain image and character of an object. A more complex process of product shaping is the combination of finished objects (Detkina and Fukin, 2010).

When designing the shape, the craftsmen first started from natural forms. Later, in the process of studying the environment, man began to establish a generalized thinking, which contributed to the development of new shapes, based on the properties of materials and their purpose.

Today, any object, even a building, can be a source of inspiration for designers. The basis for the transformation and creation of sketches of shoes was chosen architectural structure, namely the medical center Enter Architecture (https://novate.ru/blogs/ 060311/17011). The Australian design studio "Louns" has completed the construction of a modern medical and expressive complex "John Curtin school of medical research", the concept of which reflects the progressive methods of work and the desire to improve and develop technology (Fig. 1). Based on the art object, a design based on the principles of visual transformations, which are important for structuring the artistic qualities of the transformative properties of shoes, was proposed (Fig. 2).

An example of an artistic search for the shaping of the bottom of shoes on the basis of various architectural structures is shown in Fig. 3.

The complex shape of shoe parts requires a responsible approach to ensuring their performance. It is well known that the shoe in the process of wearing receives various loads that cause different types of deformation of its parts. Within the specified limits, these deformations are useful and necessary for the comfortable well-being of the shoe owner. With a rigid shoe design, a feeling of discomfort and fatigue quickly occurs. And vice versa - flexible and elastic design helps to maintain comfort for a long time. However, too flexible shoes also quickly cause fatigue, and insufficient fixation of the foot can cause injury. In addition, in the event of significant deformations, stresses may occur in the materials of shoe parts that exceed their strength and cause the destruction of these parts. Therefore, at the stage of designing shoes, their strength calculation is very important. 

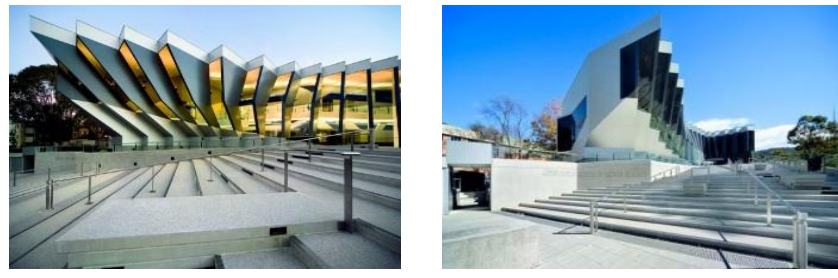

Figure 1. Art object Enter Architecture
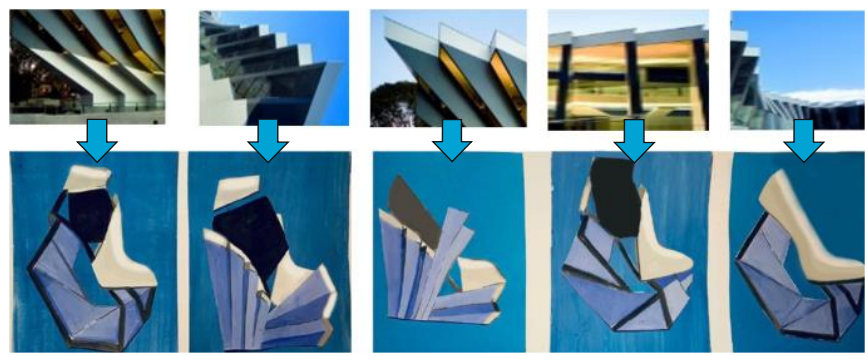

Figure 2. Elements of transformation that were used in modeling of shoes
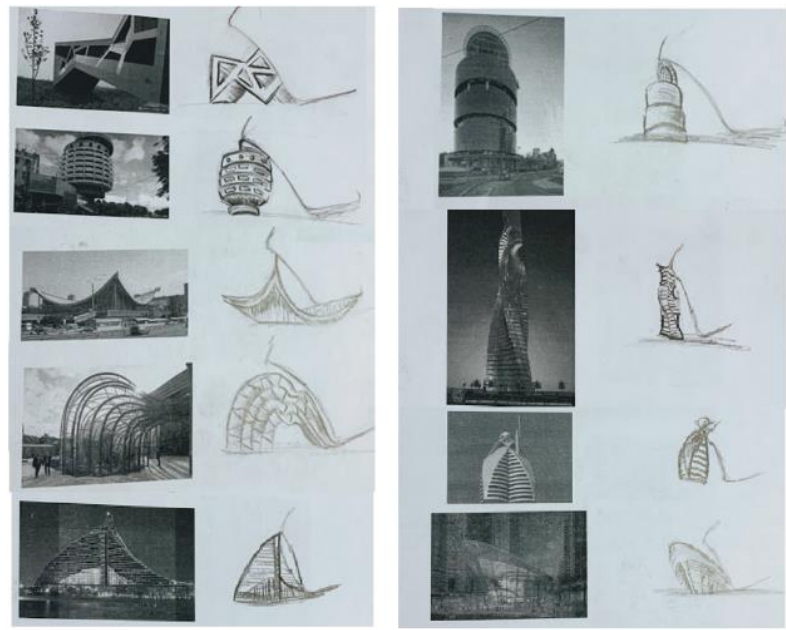

Figure 3. Transformation of art objects into shoes construction

Typical types of deformation of parts traditionally include: bending, shear, torsion, tension and compression.

The relationship between strain and normal stresses in a material is established by Hooke's law (Pisarenko et al., 2004). In the general case, this dependence has the form: $\sigma=E \varepsilon$,

where $\sigma$ - is the stress; $\varepsilon$ - is the relative deformation; $E$ - is the modulus of elasticity of the material (Young's modulus).

The calculation of polymer parts of shoes is complicated by the fact that they are not elastic bodies, and for them cannot be applied with high accuracy classical methods of resistance of materials and the theory of elasticity (Tager, 2007). For such elements, you 
can use the function of the relationship between deformation and normal stresses in polymeric materials in the form (Lebedev, 1991):

$\sigma^{m}=E \varepsilon$,

where $m$ - is the exponent, which varies from 0.6 to 1 depending on the type of material (at $m=1$, the body exhibits elastic properties, which is characteristic of metals).

In the process of designing shoe parts, you can start from the conditions of providing strength or providing the necessary rigidity. Each element can be represented as a simple part - a rod, a plate or a tube. This will make it possible to use known engineering methods of calculation taking into account the properties of the materials from which these elements of footwear are made. For example, in Kulik, 2017a, we obtained expressions for the calculation of cantilevered elements of the polymer shoe bottom under the action of a distributed load (Fig. 4, a), and Kulik, 2017b presented a method for calculating the polymer elements of the rod shape during torsion (Fig. 4, b).

The required strength and rigidity of the part can be ensured by choosing its rational geometric parameters, because the physical and mechanical properties of the already selected material cannot be influenced. Such parameters can be the thickness $h$ or radius $r$ of the part.

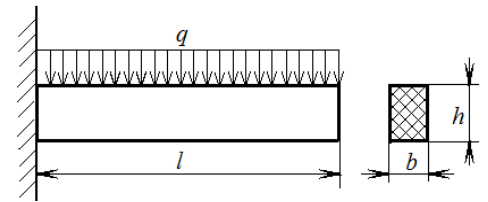

a)

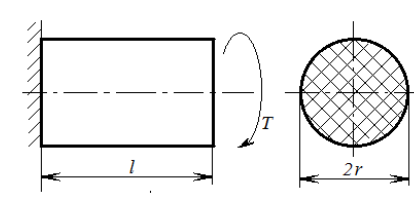

b)

Figure 4. Calculation schemes for the study of the stress-strain state of cantilevered polymer elements of footwear: $a$ - under the action of a uniform load; $b$ - when twisting

When cantilever bending of a part of rectangular cross section $b \times h$, loaded with distributed force (see Fig. 4, a), the required strength of the part is provided by the thickness determined from the expression:

$h=\sqrt{q \cdot l^{2} \cdot(1 / m+2) /(b \cdot[\sigma])} \cdot$

The condition of rigidity will be:

$$
h=2 \cdot \sqrt{\frac{\frac{1}{m}+z}{2^{m+1} \cdot m \cdot b \cdot E \cdot\left[\theta_{\max }\right]}}
$$

or

$$
h=2 \cdot \sqrt[\frac{1}{m}+2]{\frac{q^{m} \cdot l^{2 m+n} \cdot\left(\frac{1}{m}+2\right)}{2^{m+2} \cdot(m+1) \cdot b \cdot E \cdot\left[y_{\max }\right]}} .
$$

In the given expressions: $b, h, l$ - respectively width, thickness and length of the part; $q$ - is the magnitude of the load; $m$ - is the exponent that characterizes the elastic properties of the polymeric material; $[\sigma]$ - the strength of the material of the part; $[\theta]-$ angle of rotation; $[y]$ - deflection.

For the part of the rod shape shown in the diagram of Fig. 4, b, the minimum radius is determined at which the appropriate strength will be provided:

$$
r=\sqrt[3]{(T /[\tau]) \cdot(1 / m+3)}
$$

or required rigidity: 
$r=\frac{1}{m}+3 \sqrt{\frac{T \cdot l^{1 / m} \cdot(1 / m+3)}{\sqrt[m]{G \cdot[\varphi]}}}$.

In the given expressions: $r, l$ - radius and length of the rod element; $T$ - torque; $m$ - is the exponent that characterizes the elastic properties of the polymeric material; $[\tau]-$ allowable tangential stresses for the material; $[\varphi]$ - allowable angle of rotation; $G$ - is the shear modulus. To date developed a large number of different software that simplifies the process of designing shoes, to receive materials cutting schemes, to investigate manufacturing processes, to model behavior of footwear parts of various loadings.

In this study, the software product Rhinoceros 3D was used for automated design of shoe uppers and bottoms. In fig. 5 a sketch of the developed shoes is shown.

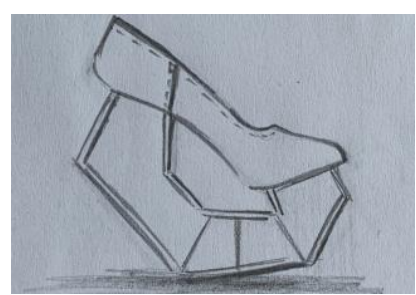

Figure 5. Sketch of shoes

SolidWorks software was used to analyze the loads that will occur in the elements of the shoe while wearing it. Fig. 6 illustrates the uneven distribution of pressure on the surface of the sole, taking into account the angle of the foot. Fig. 7 presents the analysis of stresses and strains in the sole of the shoe during operation.

As a result of modeling, it was found that the weakest part of the structure is its middle section. It is this area that receives the greatest deformation loads. To strengthen it, it was proposed to use an additional element of reinforcement, which is shown in Fig. 8. The finished product obtained by the developed project is presented in Fig. 9.

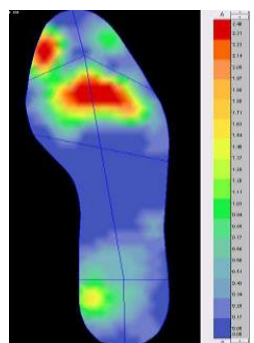

Figure 6. Weight distribution on the insole of shoes

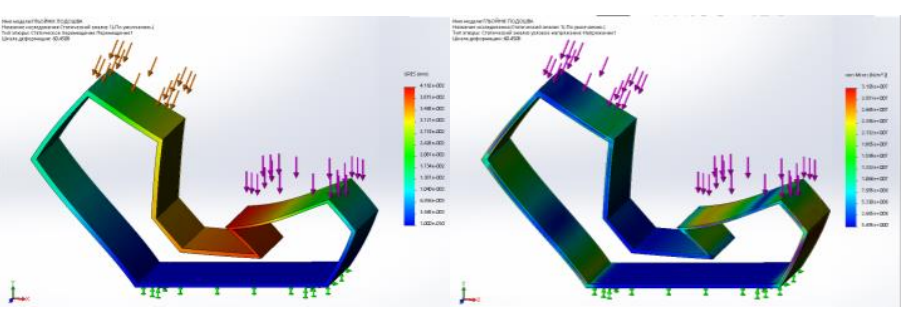

a)

Figure 7. Distribution of stresses $(a)$ and deformations $(b)$ arising in the sole of the shoe under the action of operating loads

A feature of the bottom of the developed shoes is the presence of a platform: the design of the classic platform has been transformed into a geometric object that has not only a functional purpose, but also acts as a decor. The model is based on contrasting colors that give the shoes a more aesthetic, brighter look. All details of the top are made of natural materials. The insole assembly consists of a main insole, a half insole and a metal insole; padding, which fills the space between the contours of the lasting edge of the lasted shoes. The method of forming the workpiece is external lasting and force lasting. The platform is made by a method of stamping from the rolled metal tape $2 \mathrm{~mm}$ thick (ST-3 steel). 


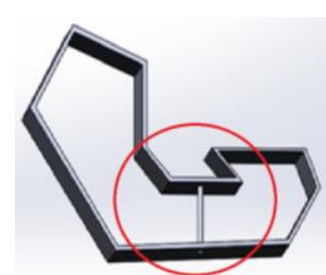

Figure 8. The element of structural reinforcement

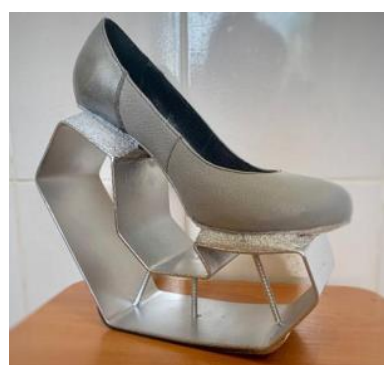

Figure 9. The made specimen

\section{CONCLUSIONS}

The study considered the methods of visual transformations that are important for structuring the artistic qualities of shoes. The used technique allows shaping a wide range of various prototypes of footwear, using a small number of transformed products. And the combination of the given techniques with modern high-tech production helps to save means of the manufacturer and to increase the service life of footwear.

Given that the complexity of structures and the emergence of new three-dimensional solutions pose more complex technical challenges for designers to implement design projects, the possibility of calculating the strength and dimensional stability of products of complex geometric shapes was analyzed taking into account the characteristics of the materials from which they are made.

Possibilities of the modern software for designing of footwear and modeling of its behavior in the course of operation are considered. Determining the most loaded areas made it possible to propose measures to improve the design without the manufacture and study of an experimental sample, which is of great importance in the manufacture of shoes of non-standard models.

As a result of the performed work, women's shoes of the "shuttle" design with an over seam back allowance were developed. Given the fact that the shoes should be comfortable and meet ergonomic indicators, the materials, the shape of the pad, and the design of the model were selected. The purpose of this shoe is everyday wearing, focused on the youth category of consumers.

\section{REFERENCES}

Detkina, D.N. and Fukin, V.A. (2010), The use of illusions of visual perception in the artistic modeling of footwear, IIC MGUDT, Moscow.

Kulik, T.I. (2017a), "Investigation of the stress-strain state of cantilevered elements of the polymer bottom of shoes under the action of distributed load", Bulletin of the Kyiv National University of Technology and Design, Technical Sciences Series, № 6 (116), pp. 66-73.

Kulik, T.I. (2017b), "Method of calculating the rod elements of the bottom of the shoe during torsion", Bulletin of Kherson National Technical University, № 1, pp. 130-135.

Lebedev, V.S. (1991), Technological processes of machines and apparatus in the production of consumer services, Legprombytizdat, Moscow.

Pisarenko, G.S., Kvitka, O.L. and Umansky, E.S. (2004), Resistance of Materials, Higher School, Kyiv.

Tager, A.A. (2007), Physical chemistry of polymers, Scientific world, Moscow.

Ustin, V.B. (2007), Composition in design, AST: Astrel, Moscow.

Zaeva-Burdonskaya, E.A. and Kurasov, S.V. (2008), Shaping in environmental design. Stylization method, MGHPU: SG Stroganov, Moscow.

*** Assortment of leather shoes, https://buklib.net/books/28806/.

*** Expressive architecture of the Australian Medical Center, https://novate.ru/blogs/060311/17011/. 